\title{
A prospective study of vasculitis patients collected in a five year period: evaluation of the Chapel Hill nomenclature
}

\author{
Søren Freiesleben Sørensen, Ole Slot, Niels Tvede, Jørgen Petersen
}

\begin{abstract}
Objective-To test the usefulness of the Chapel Hill nomenclature, supplemented with surrogate parameters, as diagnostic criteria for primary vasculitides.

Methods-To prospectively evaluate vasculitis patients according to a standardised clinical and para-clinical programme. In accordance with the Chapel Hill publication surrogate parameters were used: proteinuria, haematuria and red blood cell casts (glomerulonephritis), angiographic or ultrasonic demonstration of aneurysms or stenoses (arteritis), radiological lung infiltrates or cavitations of more than one month's duration (granuloma in the lungs), bloody nasal discharge or crusts, chronic sinusitis, otitis and/or mastoiditis, bone and/or cartilage destruction, and acute hearing loss (granuloma in upper airways).
\end{abstract}

Results-The following entities were diagnosed: giant cell arteritis $(n=14)$, Takayasu arteritis $(n=1)$, polyarteritis nodosa $(n=2)$, Wegener's granulomatosis $(n=27)$, ChurgStrauss syndrome $(n=2)$, microscopic polyangiitis $(\mathbf{n}=12)$, Henoch-Schönlein purpura $(n=2)$, cutaneous leucocytoclastic angiitis $(n=37)$, and secondary vasculitis $(n=21)$. Giant cell arteritis and cutaneous leucocytoclastic angiitis were in all cases diagnosed by biopsy. Using the Chapel Hill nomenclature supplemented with surrogate parameters, only 8 of 27 patients were diagnosed with Wegener's granulomatosis, and 3 of 12 cases with microscopic polyangiitis. The number of patients in the remaining diagnostic entities were considered to few to evaluate.

Conclusions-The Chapel Hill nomenclature, supplemented with surrogate parameters, failed to act as diagnostic criteria in Wegener's granulomatosis and microscopic polyangiitis. The following diagnostic criteria are proposed for Wegener's granulomatosis: (1) Biopsy or surrogate parameter for granulomatous inflammation in the respiratory system and (2) Biopsy verified necrotising vasculitis in small to medium sized vessels or biopsy/surrogate parameter for glomerulonephritis or positive PR3-ANCA test and (3) Lack of eosinophilia in blood and biopsy samples. The following diagnostic criteria are proposed for microscopic polyangiitis: (1) Biopsy verified necrotising vasculitis in small vessels and/or glomerulonephritis with few or no immune deposits and (2) Involvement of more than one organ system as indicated by biopsy verified vasculitis in small to medium sized vessels or surrogate parameter for glomerulonephritis and (3) Lack of biopsy and surrogate parameter for granulomatous inflammation in the respiratory system. Using these criteria all Wegener's patients and 9 of 12 patients with microscopic polyangiitis could be diagnosed.

(Ann Rheum Dis 2000;59:478-482)

Since the first attempt to classify vasculitis by Zeek, ${ }^{1}$ a great number of investigators have tried to improve classification criteria. For references see Lie. ${ }^{2}$

The most recent proposal was made at the Chapel Hill conference in North Carolina in 1992, where a nomenclature defining 10 primary vasculitides was formulated. ${ }^{3}$ The Chapel Hill Group made "great efforts to adopt names and definitions already widely accepted". However, it differed fundamentally from other classification schemes in that classic polyarteritis nodosa (PAN) was restricted to necrotising inflammation of medium sized and small arteries only without glomerulonephritis or vasculitis in arterioles, capillaries or venules. Furthermore, IgA dominant immune deposits were required in the definition of HenochSchönlein purpura, and cryoglobulin immune deposits were required for defining essential cryoglobulinaemic vasculitis. In the Chapel Hill description of Wegener's granulomatosis (WG) it was mentioned that necrotising glomerulonephritis was common, and that in microscopic polyangiitis (MPA) necrotising glomerulonephritis was very common. Thus, the presence of glomerulonephritis was not a necessary requirement in the definition of these diseases (table 1).

Even though it was emphasised that WG, Churg-Strauss syndrome and MPA are associated with ANCA, the presence of ANCA was not included in the definition of any of these three diseases.

Although the Chapel Hill report emphasised that the aim was to create a nomenclature of systemic vasculitides, we felt that the proposals might serve as diagnostic guidelines in the daily clinic, because of the clear definition of the type of vessel involved, and the presence or absence of granulomatous inflammation in the respiratory system, eosinophilia, and immune deposits in vessels. 
Table 1 The Chapel Hill nomenclature

Large vessel vasculitis

Giant cell (temporal) arteritis

Takayasu arteritis

Medium sized vessel vasculitis

Polyarteritis nodosa

Kawasaki disease

Small vessel vasculitis

Wegener's granulomatosis

Churg-Strauss syndrome

Microscopic polyangiitis

Henoch-Schönlein purpura

Essential cryoglobulinaemic vasculitis

Cutaneous leucocytoclastic angiitis
Granulomatous arteritis of the aorta and its major branches, with a predilection for the extracranial branches of the carotid artery. Often involves the temporal artery. Usually occurs in patients older than 50 and often is associated with polymyalgia rheumatica.

Granulomatous inflammation of the aorta and its major branches. Usually occurs in patients younger than 50 .

Necrotising inflammation of medium sized or small arteries without glomerulonephritis or vasculitis in arterioles, capillaries, or venules.

Arteritis involving large, medium sized, and small arteries, and associated with mucocutaneous lymph node syndrome.

Coronary arteries are often involved. Aorta and veins may be involved. Usually occurs in children.

Granulomatous inflammation involving the respiratory tract, and necrotising vasculitis affecting small to medium sized vessels (that is, capillaries, venules, arterioles, and arteries). Necrotising glomerulonephritis is common.

Eosinophil rich and granulomatous inflammation involving the respiratory tract, and necrotising vasculitis affecting small to medium sized vessels, and associated with asthma and eosinophilia.

Necrotising vasculitis, with few or no immune deposits, affecting small vessels (that is, capillaries, venules, or arterioles).

Necrotising arteritis involving small and medium sized arteries may be present. Necrotising glomerulonephritis is very

common. Pulmonary capillaritis often occurs.

Vasculitis, with IgA dominant immune deposits, affecting small vessels (that is, capillaries, venules, or arterioles). Typically

involves skin, gut, and glomeruli, and is associated with arthralgias or arthritis.

Vasculitis, with cryoglobulin immune deposits, affecting small vessels (that is, capillaries, venules, or arterioles), and associated

with cryoglobulins in serum. Skin and glomeruli are often involved.

Isolated cutaneous leucocytoclastic angiitis without systemic vasculitis or glomerulonephritis.

Modified from reference 3.

Table 2 Surrogate parameters for vasculitis

\begin{tabular}{ll}
\hline Organ involvement & Surrogate parameters \\
\hline $\begin{array}{l}\text { Glomerulonephritis } \\
\text { Arteritis }\end{array}$ & $\begin{array}{l}\text { Proteinuria and haematuria with red blood cell casts } \\
\text { Angiographic or ultrasonic demonstration of aneurysms or stenoses in arteries provided the } \\
\text { patient demonstrated other signs of vasculitis }\end{array}$ \\
$\begin{array}{l}\text { Granulomatous inflammation } \\
\text { in lower airways }\end{array}$ & $\begin{array}{l}\text { Radiologically demonstrated pulmonary infiltrates or cavitations of more than one month's } \\
\text { duration provided that all other causes such as infections and malignancies were ruled out }\end{array}$ \\
$\begin{array}{l}\text { Granulomatous inflammation } \\
\text { in upper airways }\end{array}$ & $\begin{array}{l}\text { Bloody nasal discharge and/or crusting of more than one month's duration; chronic sinusitis, } \\
\text { otitis and/or mastoiditis (proved by radiograph, CT or MRI); cranial bone and/or cartilage } \\
\text { destruction; acute hearing loss, without signs of traumatic disease }\end{array}$ \\
\hline
\end{tabular}

Furthermore, the nomenclature may be useful because it was proposed that certain symptoms and findings could be used as surrogate parameters - that is, the presence of proteinuria, haematuria, and red blood cell casts, which suggests glomerulonephritis, and certain angiographic findings as evidence for vasculitis in the involved vessels. The method used in this study was to prospectively evaluate vasculitis patients according to a standardised procedure. The aim was to test the usefulness of the Chapel Hill nomenclature, supplemented with surrogate parameters, as diagnostic criteria in the daily clinic.

\section{Methods}

The patients were prospectively included from three Copenhagen university hospitals in the period 1993-1997. Patients were recruited from the Copenhagen municipal area and from several adjacent counties. Most patients were tertiary referrals from other specialists and all referrals were included in the study.

All patients underwent standardised clinical and paraclinical examinations with emphasis on possible concurrent connective tissue disease, presence of infections, or exposure to drugs.

The ANCA test was performed with an immunofluorescence technique with subsequent testing for PR3-ANCA and MPOANCA by ELISA. ${ }^{4}$ In both ELISAs, values $\geqslant$ $10 \mathrm{U} / \mathrm{ml}$ were considered abnormal. All sera positive for C-ANCA by immunofluorescence test were PR3-ANCA positive.

Biopsy specimens from affected organs were obtained, although it was not always feasible for practical reasons.
The surrogate parameters used are shown in table 2. The surrogate parameters that indicated granulomatous inflammation in the respiratory system were adapted from the scoring systems created by the Birmingham Vasculitis Group. ${ }^{56}$

All patients were followed up for two to seven years by the respective hospitals, and the final clinical diagnosis was made in collaborative effort with the authors without regard to the presence or absence of specific criteria. ${ }^{7}$

The study was approved by the local ethics committee.

\section{Results}

Table 3 shows the number of patients in each diagnostic category. Ninety seven patients had primary vasculitis and 21 patients had secondary vasculitis.

All patients with suspected vasculitis were included prospectively. After inclusion of 14 patients with giant cell arteritis that uniformly

Table 3 Number of patients in each diagnostic category

Primary vasculitis ( $n=97$ )

Giant cell arteritis

Takayasu arteritis

Polyarteritis nodosa

Kawasaki disease

Wegener's granulomatosis

Churg-Strauss syndrome

Microscopic polyangiitis

Henoch-Schönlein purpura

Essential cryoglobulinaemic vasculitis

Cutaneous leucocytoclastic angiitis

Secondary vasculitis $(n=21)$

Rheumatoid arthritis

Systemic lupus erythematosus

Sjögrens syndrome

Waldenstroms macroglobulinaemia

Hepatitis C viraemia 
Table 4 Findings in patients with Wegener's granulomatosis

\begin{tabular}{|c|c|c|c|c|c|c|c|c|c|c|c|c|c|c|c|c|c|c|c|c|}
\hline \multirow{2}{*}{$\begin{array}{l}\text { Patient } \\
\text { number }\end{array}$} & \multirow{2}{*}{ Age } & \multirow[b]{2}{*}{ Sex } & \multicolumn{4}{|c|}{ Kidneys } & \multicolumn{2}{|l|}{ Skin } & \multicolumn{4}{|c|}{ Lungs } & \multicolumn{4}{|l|}{$E N T$} & \multicolumn{3}{|c|}{ Other organs } & \multirow[b]{2}{*}{$A N C A$} \\
\hline & & & Sym & Sur & Comment & Biop & Sym & Biop & Sym & Sur & Comment & Biop & Sym & Sur & Comment & Biop & Sym Sur & Comment & Biop & \\
\hline 2 & 65 & $M$ & + & & protu. & + & + & + & + & & cough & & + & + & bl.nas.dis. & & & & & PR3 \\
\hline 11 & 65 & $\mathrm{~F}$ & + & & ery. & & + & + & + & + & fix.infil $>1 \mathrm{mo}$ & + & + & + & sinusit/mast. & & & & & $\mathrm{MPO}$ \\
\hline 21 & 20 & $\mathrm{~F}$ & + & + & cyl.ery.protu. & + & & & + & + & fix.infil $>1 \mathrm{mo}$ & & + & + & sinusit & + & & & & PR3 \\
\hline 22 & 34 & M & + & & ery. & & + & + & + & + & fix.infil $>1 \mathrm{mo}$ & & + & + & bl.nas.dis. & & & & & PR3 \\
\hline 24 & 76 & $\mathrm{~F}$ & + & & protu. & + & & & + & + & fix.infil $>1 \mathrm{mo}$ & & & & & & & & & PR3 \\
\hline 33 & 41 & $M$ & + & & protu. & + & & & + & + & fix.infil $>1 \mathrm{mo}$ & & + & + & sinusit & & & & & PR3 \\
\hline 35 & 59 & $M$ & + & & ery. & + & + & & & & & & + & + & crusting & & + & mononeur. & & PR3 \\
\hline 36 & 56 & $M$ & & & & & & & & & & & + & + & otitis & & & & & PR3 \\
\hline 37 & 46 & $\mathrm{~F}$ & + & & ery. & & & & + & & haemoptysis & & + & + & sinusit & + & & & & PR3 \\
\hline 45 & 75 & $\mathrm{~F}$ & + & & protu. & + & & & + & + & fix.infil $>1 \mathrm{mo}$ & & + & & pur.nas.dis. & & & & & PR3 \\
\hline 49 & 68 & $\mathrm{~F}$ & + & & protu. & & & & + & + & fix.infil $>1 \mathrm{mo}$ & + & + & + & sinusit & & & & & PR3 \\
\hline 63 & 57 & $\mathrm{~F}$ & & & & & & & + & + & fix.infil $>1 \mathrm{mo}$ & + & + & + & sinusit & & & & & PR3 \\
\hline 69 & 63 & M & + & + & cyl.ery.protu. & + & & & & & & & + & + & bl.nas.dis./crust & & + & mult.infarcts & & PR3 \\
\hline 71 & 27 & $\mathrm{~F}$ & & & & & + & & + & + & caverns $>1 \mathrm{mo}$ & & & & & & & & & PR3 \\
\hline 75 & 28 & $\mathrm{~F}$ & & & & & & & + & + & caverns $>1 \mathrm{mo}$ & & + & + & sinusit & + & & & & PR3 \\
\hline 77 & 29 & $M$ & + & & protu. & + & + & & & & & & + & + & ac.h.loss & & & & & PR3 \\
\hline 82 & 23 & $\mathrm{~F}$ & & & & & + & & & & & & + & + & mastoiditis & & & & & PR3 \\
\hline 84 & 31 & M & + & + & cyl.ery.protu. & + & & & & & & & + & + & sinusit & & & & & PR3 \\
\hline 93 & 76 & $\mathrm{~F}$ & & & & & & & & & & & + & + & saddlenose & & & & & PR3 \\
\hline 97 & 67 & $\mathrm{M}$ & & & & & & & + & + & fix.infil $>1 \mathrm{mo}$ & + & + & & pur.nas.dis. & & & & & PR3 \\
\hline 98 & 62 & $\mathrm{~F}$ & & & & & + & & & & & & + & + & sinusit & & & sural nerve & + & PR3 \\
\hline 99 & 72 & $\mathrm{~F}$ & & & & & + & + & & & & & + & + & sinusit & + & & & & PR3 \\
\hline 106 & 50 & $\mathrm{M}$ & & & & & + & + & + & + & fix.infil $>1 \mathrm{mo}$ & & + & + & crusting & & + & mononeur. & & PR3 \\
\hline 120 & 52 & $\mathrm{~F}$ & + & & protu. & + & + & & + & + & caverns $>1 \mathrm{mo}$ & & + & + & saddlenose & & & portio & + & PR3 \\
\hline 121 & 38 & $M$ & + & & protu. & + & + & & + & + & fix.infil $>1 \mathrm{mo}$ & & + & + & bl.nas.dis. & & & & & PR3 \\
\hline 122 & 68 & $\mathrm{~F}$ & & & & & & & + & + & caverns $>1 \mathrm{mo}$ & + & & & & & & & & PR3 \\
\hline 123 & 78 & $\mathrm{~F}$ & & & & & + & + & + & + & caverns $>1 \mathrm{mo}$ & + & & & & & & & & NEG \\
\hline
\end{tabular}

Sym: symptoms, Sur: surrogate, Biop: biopsy, PR3: proteinase -3, MPO: myeloperoxidase, NEG: negative, protu.: proteinuria, ery.: erythrocyturia, cyl.: cylindruria, fix. infil: fixed infiltrates, bl.nas.dis.: bloody nasal, discharge, sinusit: sinusitis, mast.: mastoiditis, pur.nas.dis.: purulent nasal discharge, crust: crusting, ac.h.loss: acute hearing loss, mononeur.: mononeuritis, mult.: multiple. Biop + indicates for kidneys: necrotising glomerulonephritis, skin: leucocytoclastic vasculitis, lungs: granulomatous inflammation, ENT: granulomatous inflammation, other organs: necrotising vasculitis.

fulfilled the nomenclature it was decided to stop inclusion of more patients with giant cell arteritis.

The diagnosis of giant cell arteritis were in all cases based on characteristic biopsy findings. It is well known that in approximately $10-30$ per cent of patients with symptoms and signs of temporal arteritis cannot be demonstrated by temporal artery biopsy. ${ }^{8}$ In this study no attempts were made to assess the number of patients with negative biopsy.

The organ involvements, the results of biopsies, and the presence of surrogate parameters in 27 patients with WG are shown in table 4 . According to the Chapel Hill nomenclature the diagnosis of WG requires the demonstration of granulomatous inflammation of the respiratory tract, and necrotising vasculitis affecting small to medium sized vessels. Granulomatous inflammation of the respiratory system was demonstrated by surrogate parameter in all cases, but only confirmed by biopsy in 10 cases.
Necrotising vasculitis was only biopsy verified in eight cases (patient numbers 2, 11, 22, 98, $99,106,120,123)$. However, biopsy confirmed necrotising glomerulonephritis was demonstrated in 11 patients, and PR3-ANCA was positive in all but two patients.

According to the Chapel Hill nomenclature, the diagnosis of MPA, requires demonstration of necrotising small vessel vasculitis, not restricted to the skin, and without granulomatous inflammation in the respiratory system. Table 5 shows the organ involvement, the results of biopsies, and presence of surrogate parameters in 12 patients who were considered to have MPA. Only three patients (patient numbers $34,85,95$ ) had small vessel vasculitis demonstrated extracutaneously, thus fulfilling the Chapel Hill nomenclature. However, in nine patients glomerulonephritis was demonstrated by biopsy or surrogate parameter. In patients numbers 89 and 94 the occurrence of

Table 5 Findings in patients with microscopic polyangiitis

Microscopic polyangiitis ( $n=12$ )

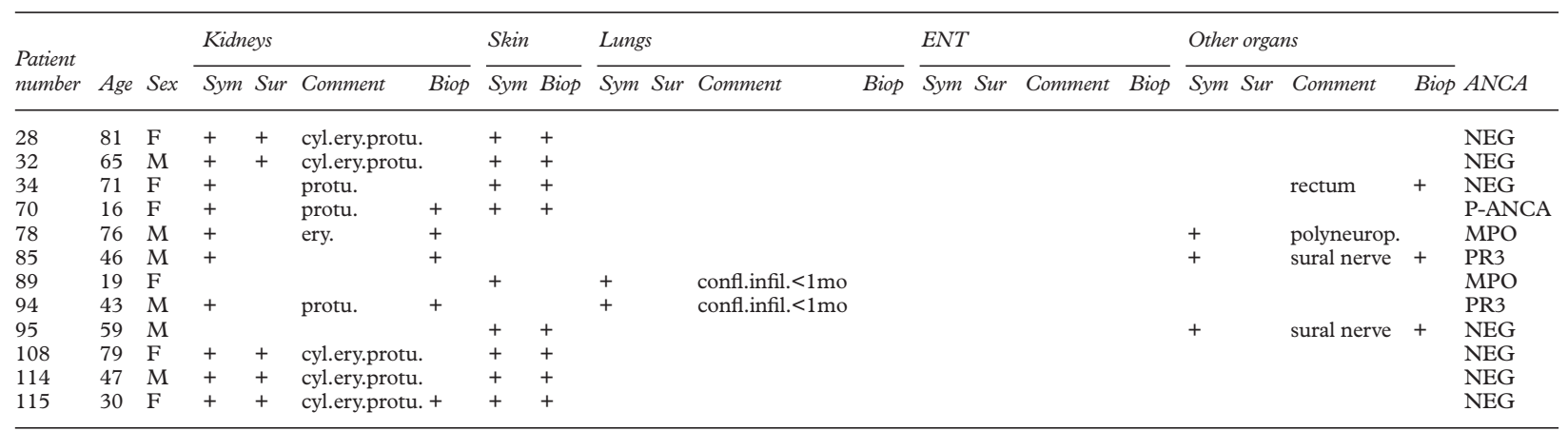

Confl.infil.: confluating infiltrates, polyneurop.: polyneuropathy. Other abbreviations as for table 4 . 
Table 6 Vasculitis in patients with cryoglobulins in blood

\begin{tabular}{|c|c|c|c|c|c|c|c|c|c|c|c|c|}
\hline $\begin{array}{l}\text { Patient } \\
\text { number }\end{array}$ & Age & Sex & Derm symptoms & $C R P$ & Urine & Clin.chem. & Auto antibodies & $M$-comp. & Ig increased & $C 3 d$ & Hep $C$ & Hep $B$ \\
\hline 115 & 30 & $\mathrm{~F}$ & livedo reticularis & 71 & cyl.ery.protu & 0 & 0 & IgG lamb & A & 113 & 0 & 0 \\
\hline 86 & 80 & M & ulcerations & 796 & 0 & 0 & IgM RF & IgM kappa & $M$ & 105 & 0 & - \\
\hline 17 & 42 & $\mathrm{~F}$ & palp.purp. & 125 & ery. & ASAT $\uparrow, \mathrm{LDH} \uparrow$ & IgM RF & IgG kappa & G & 170 & + & + \\
\hline 56 & 70 & M & palp.purp. & 196 & 0 & alk. Phos. $\uparrow$ & IgM RF & 0 & GAM & 112 & + & 0 \\
\hline 29 & 58 & $\mathrm{~F}$ & palp.purp. & 154 & 0 & ASAT $\uparrow$ & $\begin{array}{l}\text { IgM RF } \\
\text { ANA,SSA,SSB }\end{array}$ & 0 & GM & 133 & 0 & 0 \\
\hline
\end{tabular}

Derm: dermatological, clin.chem.: clinical chemistry, M-comp.: M-component, palp.purp.: palpable purpura. CRP normal value <94. Column: "Ig increased" shows the Ig class or classes increased. C3d normal value <67. hep C: 0 indicates absence of antibodies to hep C, + indicates antibodies and positive polymerase chain reaction to hep C. hep B: 0 indicates absence of antibodies to hep B surface antigen, + indicates antibodies to hep B surface antigen, - : not done. Other abbreviations as for tables 4 and 5 .

haemoptysis and transient lung infiltrates indicated pulmonary capillaritis.

Table 6 shows the clinical findings in five patients with cryoglobulinaemia in the blood. All patients had involvement of the skin, and in all cases leucocytoclastic vasculitis was demonstrated by biopsy. Patient number 115 had biopsy confirmed crescentic glomerulonephritis, but in no other patients could vasculitis be demonstrated in any other organs. In all cases analysis of $\mathrm{C} 3 \mathrm{~d}$ showed increased values indicating complement activation. Further analysis of these patients showed that patient number 86 suffered from Waldenström's macroglobulinaemia, and patient numbers 17 and 56 had hepatitis $\mathrm{C}$ viraemia, as demonstrated by the presence of hepatitis C RNA by polymerase chain reaction in the blood. Patient number 29 fulfilled all diagnostic criteria for systemic lupus erythematosus complicated with secondary Sjögren's syndrome. Accordingly, only patient number 115 seemed to have essential cryoglobulinaemic vasculitis. However, repeated skin biopsies and the kidney biopsy failed to show immune deposits in vessel walls. As the diagnosis of essential cryoglobulinaemic vasculitis requires the presence of cryoglobulin deposits, it was concluded that, according to the Chapel Hill nomenclature, this patient should be diagnosed as MPA.

The greatest group of 37 patients had cutaneous leucocytoclastic angiitis, as demonstrated by biopsy. Twenty nine patients had palpable purpura, whereas skin manifestations dominated by ulcers were seen in only eight patients. Seven patients presented with rashes that suggested cutaneous vasculitis, which, however, could not be confirmed by skin biopsy. The number of patients in the remaining diagnostic categories was considered too few for further evaluation.

\section{Discussion}

The great number of publications on classification of primary and secondary vasculitis demonstrate the inherent problems in this field. ${ }^{9-15}$ Furthermore, diagnostic criteria have not been worked out, and major problems regularly arise in the clinic when a diagnostic decision must be made with respect to the individual patient.

The advantage of the Chapel Hill nomenclature is its clarity and simplicity. Ten primary vasculitis syndromes are defined according to vessel size. The small vessel vasculitides are subdivided according to the presence or absence of granulomatous inflammation in the respiratory system, eosinophilia, and immune deposits in the vessel walls.

The Chapel Hill nomenclature was not intended to function as classification or diagnostic criteria. However, the suggestion that certain clinical findings could act as surrogate parameters implied to us that the Chapel Hill nomenclature could be used as diagnostic criteria in the daily clinic. The main purpose of this study was to test this hypothesis.

In the clinical evaluation of patients with suspected vasculitis it is essential to obtain representative biopsy specimens to (1) confirm the vasculitis diagnosis, (2) reveal the presence of granulomatous inflammation, and (3) assess the presence of immune deposits in the vessel walls. However, because of the segmentary and dynamic nature of the vasculitis process, it is often difficult in practice to obtain representative biopsies. Furthermore, in patients with lung involvement transbronchial or percutaneous biopsies often are not informative regarding the presence of chronic granuloma, although such biopsies may be essential for excluding neoplasia and chronic infection. In such cases open lung biopsy is clearly indicated, but regularly not feasible because of the inherent risk of this procedure in vulnerable patients with debilitating disease. Therefore, it was decided to consider longstanding lung infiltrates or cavitation as surrogate parameters for granulomatous inflammation in the lungs, if malignancy and infection were excluded.

Similarly, biopsies in the ENT area regularly fail to establish the presence of granulomatous inflammation, but helped ruling out malignancies and chronic infection.

Accordingly, bloody nasal discharge or crusting, chronic sinusitis, otitis and/or mastoiditis, bone and/or cartilage destruction or acute hearing loss were considered manifestations of granulomatous inflammation, if malignancy and infection could be excluded. Angiographic or ultrasonic evidence of vasculitis was accepted in patients with other signs of vasculitis in the form of general symptoms and/or organ symptoms. Finally the presence of haematuria and proteinuria and red blood cell casts was considered a sign of glomerulonephritis.

In this study the diagnosis of giant cell arteritis was accepted only if biopsy of the temporal artery had demonstrated characteristic findings. Similarly, only cases with biopsy confirmed vasculitis in the skin were included in the study. In both diseases it must be accepted 
that approximately 10-30 per cent of biopsies fail to confirm the clinical diagnosis.

With respect to the diagnosis essential cryoglobulinaemic vasculitis no patient fulfilled the criteria suggested in the Chapel Hill nomenclature. Most of the patients with vasculitis and cryoglobulinaemia in the blood were secondary vasculitides, indicating the need in such patients to perform an intensive investigation to exclude the possibility of secondary vasculitis.

Application of the Chapel Hill nomenclature supplemented with the surrogate parameters described above proved insufficient in establishing the diagnoses of WG and MPA. It appears from table 4 that the simultaneous demonstration of granulomatous inflammation that involved the respiratory tract and necrotising inflammation affecting small to medium sized vessels, was only possible in 8 of 27 patients believed to have WG.

Table 5 shows similar data for the MPA group. In only 3 of 12 cases could extracutaneous small vessel vasculitis be demonstrated in patients with no signs of granulomatous inflammation in the respiratory tract, thus fulfilling the Chapel Hill inspired diagnostic criteria.

Eleven WG patients and nine MPA patients had glomerulonephritis shown by biopsy or surrogate parameter, which in the clinical decision process was of decisive importance for the diagnosis. Furthermore, all but two of the 27 WG patients were PR3-ANCA positive, which was also of importance for the diagnostic decisions.

Our conclusion is that the Chapel Hill nomenclature, even supplemented with surrogate parameters, fails to act as diagnostic criteria in the diagnosis of WG and MPA.

We propose the following diagnostic criteria for WG:

(1) Biopsy or surrogate parameter for granulomatous inflammation in the respiratory system and

(2) Biopsy verified necrotising vasculitis in small to medium sized vessels or biopsy/ surrogate parameter for glomerulonephritis or positive PR3-ANCA test and

(3) Lack of eosinophilia in blood and biopsy samples.

It should be noted that these criteria were fulfilled by all of the patients (27 of 27) considered to have WG. At the same time, none of the patients with diagnoses other than WG fulfilled these criteria.

We propose the following diagnostic criteria for MPA:
(1) Biopsy verified necrotising vasculitis in small vessels and/or glomerulonephritis with few or no immune deposits and

(2) Involvement of more than one organ system as indicated by biopsy verified vasculitis in small to medium sized vessels or surrogate parameter for glomerulonephritis and

(3) Lack of biopsy and surrogate parameter for granulomatous inflammation in the respiratory system.

These criteria were fulfilled by 9 of 12 patients diagnosed with MPA, while none of the patients with diagnoses other than MPA fulfilled these criteria.

We are currently planning to test the diagnostic criteria proposed for WG and MPA in a prospective study where patients are collected from all major university hospitals in Denmark.

We thank Annette Møller for assistance in preparing the manuscript.

1 Zeek PM. Periarteritis nodosa: a critical review. Am J Clin Pathol 1952;22:777-90.

2 Lie JT. Nomenclature and classification of vasculitis: Plus ça change, plus c'est la même chose. Arthritis Rheum 1994;37:181-6.

3 Jennette JC, Falk RJ, Andrassy K, Bacon PA, Churg J, Gross WL, et al. Nomenclature of systemic vasculitides. Proposal of an International Consensus Conference. Arthritis Rheum 1994;37:187-92.

4 Brimnes J, Nielsen OH, Wiik A and Heegaard NHH. Autoantibodies to molecular targets in neutrophils in patients with ulcerative colitis. Dig Dis Sci 1999;44:41523 .

5 Luqmani RA, Bacon PAA, Moots RJ, Janssen BAA, Pall A, Emery $\mathrm{P}$, et al. Birningham Vasculitis Activity Score BVAS) in systemic necrotizing vasculitis. QJM 1994; 87:671-8

6 Exely AR, Bacon PA, Luqmani RA, Kitas GD, Gordon C, Savage COS, et al. Development and initial validation of the vasculitis damage index for the standardized clinical assesment of damage in the systemic vasculitides. Arthritis Rheum 1997;40:371-9.

7 Jennette JC, Falk RJ. Small-vessel vasculitis. N Engl J Med 1997;337:1512-23.

8 Bengtsson B-Å, Malmvall B-E. The epidemiology of giant cell arteritis including temporal arteritis and polymyalgia rheumatica. Arthritis Rheum 1981;24:899-904.

9 Watts RA, Jolliffe VA, Carruthers DM, Lockwood M, Scott DGI. Effect of classification on the incidence of polyarteritis nodosa and microscopic polyangiitis. Arthritis Rheum 1996;39:1208-12.

10 Scott DGI, Watts RA. Classification and epidemiology of systemic vasculitis (editorial). Br J Rheumatol 1994;33: systemic

11 Watts RA, Carruthers DM,Scott DGI. Epidemiology of systemic vasculitis: Changing incidence or definition? Semin Arthritis Rheum 1995;25:28-34.

12 Rao JK, Allen NB, Pincus T. Limitations of the 1990 American College of Rheumatology classification criteria in the diagnosis of vasculitis. Ann Intern Med 1998;129: $345-52$

13 Watts RA, Jolliffe VA, Grattan CE, Elliott J, Lockwood M, Scott DG. Cutaneuous vasculitis in a defined populationclinical and epidemiological associations. J Rheumatol 1998;25:920-4.

14 Reid AJ, Harison BD, Watts RA, Watkin SW, McCann BG, Scott DG. Churg-Strauss syndrome in a district hospital. OJM 1998;91:219-229.

15 Blanco R, Martínez-Taboada VM, Rodríguez-Valverde V, García-Fuentes $M$. Cutaneous vasculitis in children and adults. Associated diseases and etiologic factors in 303 patients. Medicine 1998;77:403-18. 